\title{
Pratiques
}

Linguistique, littérature, didactique

\section{Contribution à une analyse de l'ethos discursif de l'enseignant en formation, envisagé à travers le critère de la réflexivité}

\section{Pascale Delormas}

\section{(2) OpenEdition \\ Journals}

Édition électronique

URL : http://journals.openedition.org/pratiques/1953

DOI : 10.4000/pratiques. 1953

ISSN : 2425-2042

Éditeur

Centre de recherche sur les médiations (CREM)

\section{Édition imprimée}

Date de publication : 15 juin 2012

Pagination : 100-114

Référence électronique

Pascale Delormas, « Contribution à une analyse de l'ethos discursif de l'enseignant en formation, envisagé à travers le critère de la réflexivité », Pratiques [En ligne], 153-154 | 2012, mis en ligne le 16 juin 2014, consulté le 20 avril 2019. URL : http://journals.openedition.org/pratiques/1953 ; DOI : 10.4000/pratiques. 1953 


\title{
Contribution à une analyse de l'ethos discursif de l'enseignant en formation, envisagé à travers le critère de la réflexivité
}

\section{Pascale Delormas}

\author{
Circeft-Escol-UPEC
}

En cette période de prise en charge de la formation des enseignants par les universités, le difficile équilibre entre pratique de terrain et théorisation de la pratique risque d'être fragilisé et il semble opportun de mieux cerner à travers les écrits des différents acteurs de l'institution scolaire les modes discursifs d'incorporation du métier d'enseignant. L'objectif de cette recherche est de montrer selon quelles modalités les différentes pratiques de formation des enseignants participent à la constitution d'un ethos professionnel à travers l'examen d'une compétence qui avait été introduite massivement dans les curricula, la pratique réflexive.

On interroge ici l'ethos réflexif de l'enseignant en prenant en compte le discours en tant qu'il est une manifestation palpable de phénomènes qui touchent à l'institution. Au niveau théorique on verra ce que le point de vue de l'analyse du discours sur le discours réflexif dans la formation des maîtres peut apporter à la compréhension de l'institution scolaire.

\section{Cadre théorique, démarche et corpus}

Nous adoptons une démarche double : une démarche intégrative en vue de faire apparaître des similitudes entre des discours dispersés et une démarche analytique qui vise à débusquer les silences et les discontinuités énonciatives. L'approche intégrative articule les diverses composantes d'une formation discursive, l'approche analytique désarticule le discours pour révéler une inconsistance radicale.

La démarche « intégrative » vise à articuler les diverses composantes de l'archive à travers un quadruple jeu de relations : celui des enchaînements intra-textuels, 
celui des divers textes à l'intérieur d'une même archive, celui de l'archive dans un réseau intertextuel, celui de l'archive et de son «contexte ». ${ }^{(1)}$

Selon la conception de l'analyse du discours, l'institution n'est jamais stable. Elle revisite précisément la notion d'archive telle que la définit Foucault : l'interdiscours mobilisant et reconditionnant en permanence les textes fondateurs, il n'y a pas de thésaurus figé. Pour approcher l'interdiscours, on examine d'une part le modèle institutionnel qui exerce des contraintes sur la fonction énonciative de ses membres et d'autre part l'activité des individus isolés qui y participent, c'est-à-dire l'injonction institutionnelle et les divers positionnements qu'elle induit et qui la légitiment. En effet, selon Maingueneau (1993),

le geste inaugural de 1'AD consiste à ramener à l'unité d'un positionnement une dispersion d'énoncés. Elle se distingue d'autres disciplines par la nature du principe qui préside à ce regroupement. Pour elle ce n'est pas un critère formel, d'ordre typologique en particulier, qui doit intervenir mais le rapport à un lieu d'énonciation permettant d'identifier ce qu'on appelle à la suite de l'Archéologie du savoir de M. Foucault une "formation discursive". [...] Le plus souvent une formation discursive ne correspond pas à un genre unique mais en associe plusieurs (tracts, manifestes, articles de journaux...) [...] la diversité des genres d'une formation discursive, loin d'être un accident survenu à un noyau de sens stable, contribue à définir son identité.

Pour cette étude, on prend en compte les différents niveaux institutionnels enchâssés ${ }^{(2)}$ : le niveau national/ministériel des Programmes, le niveau académique des lieux de formation auquel est déléguée la charge de reconfigurer la prescription initiale, le niveau de la recherche scientifique dont les résultats étayent le programme des responsables de la politique de formation et les politiques d'accompagnement et d'évaluation. Les individus, c'est-à-dire les stagiaires en formation et leurs formateurs, cherchent à se conformer à cette prescription comme le manifestent les écrits de formation, lieux privilégiés de la construction d'un ethos de "praticien réflexif » au sens de Schön (1993). Il s'agit d' "écrits réflexifs », de portfolios, de journaux de bord qui les accompagnent, de mémoires professionnels - modalité de validation encore en vigueur dans certains IUFM - et bientôt des notes de recherche et des mémoires de Master des métiers de l'enseignement. Les rapports de visite de stage rédigés par les formateurs leur sont consubstantiels, en tant qu'ils en constituent le guidage et une référence normative qui renchérit sur la commande de l'institution. Les formulaires de «visite-conseil» ou de visite de validation sont en effet imposés par l'institution avec l'objectif de normaliser les modalités d'évaluation.

La démarche intégrative suppose que la légitimité des énonciateurs soit référée à la formation discursive dans laquelle ils se trouvent pris et non à une quelconque subjectivité. Ainsi, un ethos professionnel dans le cadre d'une formation discursive repose sur l'expression d'un "sentiment d'appartenance » à une collectivité dans laquelle on est intronisé, d'un rituel nécessaire à l'acculturation au métier et à sa déontologie. L'approche analytique réaliste s'efforce de saisir la

(1) D. Maingueneau (1991: 26).

(2) Cf. Oger et Ollivier-Yanniv (2003). 
spécificité d'une position discursive en cherchant les incohérences, les failles d'une unité qui ne serait qu'imaginaire.

[Par opposition à l'approche analytique représentative, elle] s'efforce de faire apparaître par un "espacement de la lecture" l'impossible discours qu'il trahit (manifeste et masque). Passent au premier plan les multiples phénomènes de polyphonie énonciative, les décalages, les glissements, les lacunes. ${ }^{(3)}$

Ainsi, nous pouvons constater une hétérogénéité des pratiques qui questionne le fonctionnement de l'institution scolaire notamment en ce qui concerne la mise en œuvre dans la formation de la notion de «praticien réflexif» et, depuis, critère dominant de l'entrée réussie dans le métier.

J'ai restreint mon corpus aux productions écrites des acteurs de deux IUFM différents (Académies de Créteil et de Paris). Il s'agit de faire émerger les caractéristiques communes et les discontinuités énonciatives de la formation discursive dont elle advient à travers la comparaison de 26 écrits réflexifs d'enseignants stagiaires et de 30 rapports de visite de stage de types différents établis par les formateurs. Le critère de recueil des données est celui de la localisation géographique. Ma décision repose sur l'hypothèse que les pratiques très contrastées que l'on constate d'une académie à l'autre sont observables à un niveau discursif. J'aurais pu choisir le critère de l'origine disciplinaire des stagiaires ou celui de la catégorie des formateurs intervenant dans la formation dont l'habitus professionnel est divers ${ }^{(4)}$. L'analyse de l'échantillonnage associe approche quantitative et approche qualitative.

\section{Analyse de l'interdiscours de l'écrit professionnel en formation des enseignants}

Il est nécessaire de considérer les recoupements et éventuelles contradictions des discours institutionnels (ministériel et académique) et des discours scientifiques qui sont censés étayer leurs décisions.

\subsection{Le discours institutionnel}

\section{La prescription ministérielle}

La preuve d'une entrée réussie dans le métier est réclamée par l'institution en fonction de normes souvent implicites auxquelles l'impétrant est tenu de se soumettre s'il veut devenir fonctionnaire. Le mémoire professionnel est envisagé

(3) Maingueneau (1993). Et puis : "La démarche "représentative" s'inscrit davantage dans le prolongement d'une lexicologie des contextes issue de la philologie qui s'efforce de saisir la spécificité d'une position discursive à travers son vocabulaire. Alors que l'approche "réaliste" traque les points d'inconsistances discursives, l'approche "représentative" cherche des points de condensation de l'identité discursive. "

(4) Prag, Maître formateur, enseignant-chercheur n'ont pas le même regard. 
comme une réponse à l'injonction institutionnelle en matière de réflexivité. Le portail des IUFM stipulait que :

le mémoire professionnel occupe une place importante dans la formation initiale des futurs enseignants, comme dans toute formation en alternance à caractère universitaire. Réalisé avec l'appui et l'accompagnement d'un directeur de mémoire, il a vocation à permettre au futur enseignant d'analyser de façon formalisée et distanciée, sa pratique professionnelle.

Et il préconisait 1' " analyse de pratiques professionnelles, dont la forme la plus aboutie est la rédaction d'un mémoire professionnel ». Les principes et les modalités d'organisation étaient fixés comme suit :

Production écrite personnelle, le mémoire professionnel associe une problématique pédagogique élaborée à des éclairages théoriques, enrichis de l'expérience auprès des élèves. Dans la mesure où il impose le recul critique, l'étayage documentaire et l'entraînement à une forme d'écriture longue, il représente l'aboutissement de la démarche réflexive développée au cours de la formation. ${ }^{(5)}$

En 2006, 1'introduction d'un référentiel de 10 compétences faisait évoluer la donne et impulsait de nouveaux dispositifs en 2007.

1. Agir en fonctionnaire de l'Etat et de façon éthique et responsable ;

2. Maîtriser la langue française pour enseigner et communiquer

3. Maîtriser les disciplines et avoir une bonne culture générale

4. Concevoir et mettre en œuvre son enseignement

5. Organiser le travail de la classe

6. Prendre en compte la diversité des élèves

7. Evaluer les élèves

8. Compétence en informatique

9. Travailler en équipe et coopérer avec les parents et les partenaires de l'école

10. Se former et innover

Ces compétences sont elles-mêmes découpées en sous-rubriques. Imposé par l'institution, le choix thématique opéré par le stagiaire ne peut alors intervenir qu'à la marge. De ce fait on pourrait s'attendre à un cadrage univoque. Nous constatons qu'il n'en est rien.

\section{La prescription académique}

Les textes de cadrage de chaque IUFM manifestent en effet une interprétation très hétérogène des textes du Ministère. Leur autonomie leur donne toute latitude pour énoncer des exigences, des modes de diffusion et de validation spécifiques : de la production d'un mémoire de type universitaire (traditionnel) à un travail qui offre toutes les apparences de l'écrit réflexif, en passant par la possibilité de choisir entre les 2 types de production (comme ce fut le cas dans l'académie d'Orléans-Tours).

Il en résulte des écrits fort différents en longueur et en contenu. Par exemple,

(5) BO encart $n^{\circ} 15-$ Circulaire $n^{\circ} 2002-070$. 
dans les portfolios de l'académie de Paris, chaque compétence fait l'objet d'un recueil de 2 documents (on attend donc 18 documents pour 9 compétences dans chaque portfolio - la compétence en informatique étant traitée à part). Ces documents doivent être décrits et commentés pour illustrer une problématique à laquelle se serait heurté le stagiaire. Dans les «écrits réflexifs » de l'Académie de Créteil, on a privilégié la centration sur la description d'une " situation de classe ", d'un objet d'enseignement et d'une ou deux compétences.

Cette situation s'explique : le discours prescriptif réfère lui-même sans explicitation à des orientations diverses de la recherche en Sciences de l'éducation quant à la définition à donner à la notion de « réflexivité ».

\subsection{Le discours scientifique}

En effet, on observe des conceptions hétérogènes de la réflexivité et de son évaluation. Schön (1993) témoigne de la difficulté à mettre en œuvre la réflexivité dont il existerait trois types:

Le premier type est une réflexion pratique effectuée avant une action afin de guider les actions à venir. Le deuxième consiste à réfléchir au moment même de la mise en œuvre d'une action alors que le troisième sert plutôt à examiner l'efficacité d'une action après coup. Or, la manière de stimuler ou déclencher ces trois types de réflexion à la formation des maîtres peut s'avérer assez complexe, d'autant plus que la réflexion n'est pas habituellement associée à l'enseignement, perçu comme une action immédiate alors que la réflexion est plutôt perçue comme une notion académique.

Le retour réflexif impliquant de capter la réflexion en cours d'action et sur l'action, il s'avère pour certains dépasser le cadre de la formation. Michaux et Alin (2011) reprennent-ils, en la réduisant à quatre niveaux d'indice réflexif(Ir), la classification de N. Hatton et D. Smith (1995) ${ }^{(6)}$ qui tient compte de la complexité de la temporalité dans laquelle s'inscrit ce type d'écriture.

Le niveau 1(Ir=1): Ecrit descriptif sans réflexion (anecdotique); description de situation, utilisation de « recette », la solution s'applique sans réflexion.

Le niveau $2(\mathrm{Ir}=2)$ : Ecrit descriptif avec jugements personnels (états d'âme); les choix sont justifiés sur des jugements personnels sans véritable analyse, commentaires personnels.

Le niveau $3(\mathrm{Ir}=3)$ : Ecrit argumentatif (décision) ; les choix sont argumentés, méthodologie justifiée, démarche cognitive (expérimentale).

Le niveau $4(\mathrm{Ir}=4)$ : Ecrit critique (distance); dite de niveau 3 avec contextes élargis (socio-culturels, historiques, politiques...), processus métacognitifs, références bibliographiques, critères éthiques ou moraux, forte cohérence globale des idées et concepts développés.

(6) Hatton et Smith considèrent la réflexion comme étant un processus cognitif délibéré mettant en relation le sujet choisi, les connaissances antérieures et les croyances de la personne. Ce qui veut dire que lorsqu'un enseignant se retrouve devant une situation difficile, il doit examiner l'information, reformuler le problème, chercher des solutions, évaluer les conséquences et prendre les actions en fonction des résultats de sa réflexion. 
Les critères d'analyse adoptés par Ricard-Fersing, Bubant-Berglin et Crinon (2002) sont différents. Avant l'introduction des écrits réflexifs dans le cursus de la formation des maîtres en France, ils ont mené un travail de comparaison de mémoires professionnels français et anglais avec des portfolios nord-américains pour observer les modes d'apparition de l'auteur dans les écrits professionnels. Ils ont montré que ces écrits, qui réfèrent à travers leur désignation à des intentions différentes, relevaient d'acceptions distinctes de la réflexivité : les portfolios nord américains mettent l'accent sur la dimension personnelle du processus de formation, les mémoires français sur la dimension professionnelle, les mémoires anglais sur la dimension scientifique en déclinant comme suit les éléments d'autoanalyse :

Personnel : Point de vue unique du stagiaire /Ordre : narration, critique, rectification, projet révisé /Déclaration de principes et valeurs/éthique/difficultés de l'enseignant et de l'élève.

Professionnel : Centration sur la préparation de la classe et décisions/ centration sur un problème unique/ pratique en situation réelle/questionnements intimes/référence théorique biblio/ présence d'annexes ${ }^{(7)}$.

Scientifique : Mise en scène du chercheur/ référence aux textes officiels/les apprentissages de l'élève comme objets de savoir, de recherche/ une démarche des références théoriques aux leçons professionnelles. La dimension spéculative scientifique est nettement valorisée.

Ricard-Fersing et alii appelaient de leurs vœux des aménagements qui auraient privilégié l'approche américaine, c'est-à-dire le modèle " personnel». Leur hypothèse était que l'introduction des «écrits réflexifs » dans la formation en France pouvait améliorer la qualité des écrits professionnels en les orientant vers un caractère plus véritablement réflexif en mettant en jeu la personne.

\section{Analyse comparée d'écrits de formation}

Il ne m'a pas semblé incongru de faire un rapprochement entre ethos du stagiaire et ethos du formateur. L'expérience personnelle est particulièrement prégnante dans les représentations que l'on se fait de son futur métier; à cette " image » se superpose celle qu' offrent collègues et formateurs. Les écrits de ces derniers sont le lieu privilégié de la manifestation d'un jugement sur la compétence réflexive du stagiaire ${ }^{(8)}$ : ce sont des comptes rendus/conseils et des comp-

(7) Le positionnement professionnel du stagiaire suppose qu'il tienne compte de la collectivité et de sa place dans celle-ci comme agent de l'état. Comme le disent Crinon et Velcic-Canivez (2006) et Achard-Bayle (2006), en passant du « je » au « on », le rapport de stage manifeste la présence d'un je autobiographique et d'un je nouvelle expérience. La gestion d'une énonciation conforme est l'objectif à atteindre et c'est ce que nous pouvons aujourd'hui évaluer. Au niveau linguistique, la tension entre je autobiographique et je professionnel qui s'exprime par l'alternance du «je » personnel et subjectif et du « on » collectif objectivant et au niveau psychologique, elle témoignerait de la difficulté à s'ouvrir à une écriture personnelle parce que ce ne serait pas d'usage dans la formation étudiante de faire appel à l'expérience.

(8) $\mathrm{BO}$ encart $\mathrm{n}^{\circ} 15-$ Circulaire $\mathrm{n}^{\circ} 2002-070$. 
tes rendus évaluatifs de séances de classe observées et de l'entretien qui s'ensuit et, une valeur formative leur étant octroyée, ils participent à plus d'un titre à la configuration de l'ethos attendu du futur professeur.

\subsection{L'ethos des futurs enseignants dans leurs écrits réflexifs}

\section{Cadre méthodologique}

Les écrits réflexifs préconisés dans les nouveaux Programmes constitueraient une initiation et une trace des progrès réalisés en matière de réflexivité. À travers l'appropriation des normes textuelles des écrits demandés par l'institution, la représentation que le futur professionnel se fait du métier est étayée par l'expérience du terrain et les retours sur les pratiques. La présence d'indices discursifs (posture, autorité et identité socio-discursive) est révélatrice de l'identité professionnelle en construction comme leur absence peut l'être d'une carence.

Nous avons recherché dans les nouveaux écrits français les caractéristiques typologiques détectées par Ricard-Fersing et alii dans les portfolios américains et dans les mémoires professionnels anglais et français pour y découvrir les éventuels changements intervenus : l'évolution de la prescription a-t-elle eu l'effet que les sociologues de l'éducation appellent de leur vœu, c'est-à-dire une évolution vers une plus grande réflexivité personnelle ? C'est ce que nous sommes en mesure d'explorer aujourd'hui : bien qu'ils ne soient pas généralisés, les nouveaux formats introduits ont fourni un nombre de productions suffisant pour permettre une étude à nouveaux frais, chantier dont je ne donne aujourd'hui qu'une première étape et cela d'autant plus que le cadre du master change la donne.

Comparaison des écrits réflexifs produits dans les académies de Créteil et de Paris

Le tableau ci-dessous fournit la synthèse d'une analyse quantitative et qualitative (thématique) dont le détail est fourni en annexe.

\begin{tabular}{|l|c|c|}
\hline \multicolumn{1}{|c|}{ Académies } & Paris & Créteil \\
\hline Postures & $\mathrm{X}$ & \\
\hline Posture scientifique & $\mathrm{X}$ & $\mathrm{X}$ \\
\hline Posture professionnelle & & $\mathrm{X}$ \\
\hline Posture personnelle & & \\
\hline
\end{tabular}

\section{Un genre routinier}

L'approche de Van Manen (1977), laquelle propose une hiérarchie de la réflexion pratique selon 3 paliers : un palier technique, un palier praxique et enfin un palier critique, semble être la formule adoptée par l'institut de formation des maîtres de l'académie de Paris. Il semble que les écrits réflexifs dans l'académie de Créteil ont adopté massivement, en sus des caractéristiques des mémoires, 
celle des portfolios américains. Parviennent-ils à une plus grande authenticité ? La question plus globale de la culture qui les sous-tend est moins décisive qu'il ne semble si l'on considère l'étude comparée menée par C. Donahue (2008).

Beaucoup de choses ont été dites et écrites à propos de l'ambiguïté qui règne dans la formation des enseignants évaluée à l'aune de la compétence réflexive. On constate que les productions des stagiaires adoptent dans tous les cas un format finalement extrêmement rigide propre à décourager toute tentative de faire émerger un véritable regard réflexif sur sa pratique. En effet, la comparaison des productions des stagiaires montre que le respect de la commande induit des écrits standardisés, qui tendent à épouser les normes et les stéréotypes en vigueur dans la communauté discursive.

Crinon et Guigne (2006) montrent la rareté des indices de réflexivité effective dans les écrits réflexifs demandés en formation des maîtres mais ils relèvent des modes de contournement et de simulation de réflexivité. Des dérives constantes montrent qu'un format de routine transite entre pairs alors même qu'il est banni par les formateurs. On a rarement autre chose que des remarques pseudo personnelles et convenues. La difficulté n'est pas véritablement analysée. Elle est toujours dominée par la nécessité d'apparaître comme conforme. Ainsi, souvent, la formulation de la critique de la formation reçue en IUFM, qui se voudrait nuancée, manque d'arguments et rate sa cible. Les fréquentes manifestations d'autosatisfaction sont inappropriées comme des formulations maladroites le montrent : «Tout au long de l'année, j'ai gardé ma volonté de m'améliorer et mes difficultés m'ont été utiles pour mesurer mes progrès, pour aller plus loin grâce à la formation reçue $»$.

On note que la centration sur la personne de l'enseignant dans l'académie de Créteil se fait au détriment de l'exigence scientifique. En revanche, la dimension personnelle est peu présente dans les productions de l'Institut de Paris. La tendance lourde est un mixte d'approche scientifique et professionnelle.

\section{La question de l'articulation théorie/pratique}

Les qualités scientifiques repérées dans les mémoires anglais ne sont pas investies, ni dans la forme ni dans les faits, dans les écrits réflexifs de Créteil. L'ethos de chercheur n'y est pas développé (pas de bibliographie, pas de référence théorique), ce qui dans le schéma national d'une formation en Master poserait problème. L'absence totale de références théoriques étonne mais comme le rappelle Perrenoud (1994), la pratique réflexive au sens où Schön et lui-même l'entendent, n'est pas une méthodologie de recherche.

Dans les portfolios de l'académie de Paris, il s'agit de mettre en scène un parcours sans faute. Les documents viennent en appoint, pour illustrer une certitude ou faire écho à une démarche qui s'est soldée en général par une réussite. L'affirmation que le contrat avec l'institution est rempli prédomine. Le rapport à l'abstraction est décelable. Les documents sont issus de la recherche mais donnent lieu à une simple application et parfois à une remise en cause argumentée et illustrée par l'exemple d'une mise en œuvre. Il est en général très peu exigeant (une référence à Hagège serait l'occurrence la plus théorique ; on peut y voir figurer un article découvert sur Internet, non signé) et lorsqu'il s'agit d'autoévaluation 
il apparaît que la notion n'est pas assimilée. L'innovation est une coquille vide comme si les futurs professeurs ne s'autorisaient pas à tenter la moindre expérience, sans doute par souci de conformité. On peut prolonger la réflexion de Perrenoud qui prétend que « décomposer les compétences peut les faire disparaître...» en se demandant dans quelle mesure un écrit peut véritablement être qualifié de « réflexif» si les thèmes en sont déjà dictés, comme c'est le cas dans l'académie de Paris. Cette situation de double contrainte donne lieu à des maladresses relevées dans de nombreux écrits qui ne font que répéter une parole institutionnelle et avaliser une norme sans en reconstruire le caractère nécessaire. Ainsi, on lit trop souvent cette formule : «Cette compétence est un élément fondamental de l'éducation et du professeur des écoles».

\subsection{L'ethos du formateur dans les rapports de visite}

L'exposé de cette analyse sera beaucoup plus bref étant donné le format de l'article et je n'en fournis que les conclusions. J'ai restreint mon corpus à des écrits produits par les formateurs des deux mêmes académies d'île de France. J'ai le projet d'en donner une image plus complète et plus nuancée en augmentant le panel des données qui contribuent à renforcer l'appartenance au corps de l'école (plus d'académies, davantage d'écrits, observation de discours produits dans les communautés dites virtuelles, blogs et forums d'enseignants).

Deux caractéristiques de la voix du formateur dans l'écrit de formation peuvent faire l'objet d'une analyse en matière de réflexivité.

1) L'analyse des postures des stagiaires réclame, comme le répètent les sociologues, que l'impact de la présence des coénonciateurs dans l'interaction ne soit pas occulté. Etant donné la prégnance de l'effet miroir, la vigilance du formateur doit être accrue et l'identification des espaces spécifiques et des rôles de l'un et de l'autre des partenaires doit faire l'objet d'un soin tout particulier.

2) Étant donné la forte mobilisation du modèle dans la transmission, on pourrait attendre, en toute cohérence, qu'ils adoptent de façon exemplaire la posture réflexive qu'ils sont censés évaluer : les formateurs partagent la même activité que ceux qu'ils accompagnent et à qui ils sont censés transmettre les bonnes pratiques. Ce deuxième aspect n'est toutefois pas prescrit par l'institution.

\section{Analyse des postures réflexives de l'énonciateur}

Les marques d'une métaréflexivité, réflexivité en abyme, que l'on pourrait s'attendre à voir développer dans des rapports de visite sont absentes. J'ai pu constater que le rapport de visite entretient l'image d'un rapport professionnel réussi entre stagiaire et formateur (que ce soit dans la critique ou dans l'éloge). Le formateur adopte une posture fondamentalement institutionnelle et prescriptive sans que jamais son rôle et sa propre posture ne soient interrogés.

Le compte rendu de l'observation et de l'entretien qui lui succède - peut-être parce qu'il est perçu par le formateur comme un document à simple valeur administrative - ne prend jamais en considération la dimension de l'interaction trian- 
gulaire entre terrain, formateur et stagiaire. Par exemple, lorsque les formes personnelles apparaissent, c'est pour modaliser un discours rapporté : « je lui conseille»; la forme impersonnelle du « on » est privilégiée ; on ne rencontre jamais d'adresse en « vous » sinon dans 2 des 30 rapports. Des formulations neutralisantes comme « Mademoiselle X, la stagiaire,... » apparaissent dans tous les rapports.

L'ethos du professionnel de l'enseignement dont la pratique serait étayée par la recherche n'est pas davantage visible dans les discours des formateurs que dans ceux des stagiaires. La dimension scientifique n'apparaît qu'exceptionnellement, sous la forme de conseils bibliographiques. L'articulation des pratiques avec la recherche en est absente, comme si cet écrit n'en était pas le lieu. Homologie avec la posture du stagiaire, prégnance du terrain et urgence de la situation seraient sans doute des explications plausibles à une telle attitude.

\section{Écrit non institutionnel et évaluation de la formation professionnelle}

Dans l'académie de Créteil l'obligation de remettre à l'administration un document attestant de la visite effectuée au stagiaire a été introduite tardivement: les formateurs n'en ont renseigné que les deux dernières années précédant l'introduction du Master. Cependant, ils ont maintenu la première forme scripturale que prenait le témoignage de leur passage dans le lieu de stage. Le stagiaire recevait du formateur un billet manuscrit (en général) dont le principe consensuel reposait sur une évaluation sur le vif assortie de conseils personnalisés.

Cet écrit non institutionnel est inscrit dans l'habitus local ; loin d'être anecdotique son existence est révélatrice à rebours du statut de l'institution. Comment interpréter ce contournement paradoxal inscrit dans les pratiques des formateurs? L'analyse de Wrana (2006) nous y aide : si l'on considère, comme elle nous y incite, que la faible implication des stagiaires est imputable à une résistance à la marchandisation générale de la formation et à la flexibilité accrue des futurs agents de l'éducation à qui incombe la totale responsabilité de leurs actes, alors l'écrit qui promeut l'évitement du point de vue administratif aurait une certaine capacité de formation; elle me semble s'apparenter au compagnonnage tant décrié aujourd'hui. Il échappe au contrôle - c'est là sans doute son intérêt aux yeux des formateurs qui y étaient très attachés - et ... à l'analyse du discours.

\section{Bilan et questions ouvertes}

On a vu dans notre étude que l'intervention locale (académique) battait en brèche l'illusion d'homogénéité de la formation et que la prescription d'une posture réflexive tentait de concilier les deux objectifs paradoxaux de la reproduction et de la subjectivité, de la socialisation et de la constitution de l'individu en sujet. Il apparaît que l'ethos réflexif promu est complexe et peu homogène. Le geste réflexif attendu des stagiaires est loin d'être personnel : il livre une forme « dégradée d'investissement » étant donné les formes stéréotypées qu'elles prennent. Mais l'ethos du formateur l'est également.

On a pu constater que les écrits professionnels gagnent à être analysés comme 
éléments d'un interdiscours, c'est-à-dire inscrits dans un espace-temps et participant d'une coénonciation dont les partenaires qu'ils soient évoqués ou non dans les écrits professionnels (interaction implicite) ne doivent pas être écartés. Les représentations des formateurs et des stagiaires quant à la formation idoine reflètent tout autant qu'elles induisent diverses conceptions de la réflexivité selon un cercle vertueux dont on ne saisit ni la cause ni l'effet. Le cadrage national (centralisation oblige) laisse, comme on l'a vu, une marge de « liberté » considérable. On peut l'envisager autrement que comme une coquille vide à investir de façon variée : les productions d'écrits professionnels réflexifs sont à articuler à l'idéologie qui en suscite la variété et les rend possibles ${ }^{(9)}$.

La nécessité d'un regard réflexif qui développe la dimension personnelle apparaît comme davantage nécessaire dans les départements de la banlieue que dans la ville de Paris ; de même on semble considérer dans la formation des parisiens que la spéculation intellectuelle serait accessible aux candidats et utile aux maîtres. Ce qui est hautement problématique, ce n'est pas seulement l'écart entre les représentations et la réalité de terrain, mais le fait que les représentations orientent à ce point des options de formation. Par exemple, minorer la dimension scientifique de la formation au nom de nécessités qui seraient plus urgentes (on pense par exemple à la gestion de classe) est problématique dans la mesure où ce choix est fondé sur une interprétation de la théorie et du rapport au savoir des futurs enseignants susceptible d'entraver la modification souhaitable des rapports à l'intérieur de l'école comme lieu d'apprentissage tout autant que comme lieu d'épanouissement. La réflexivité comme la conçoit Schön est souvent réduite à ce qu'elle n'est pas c'est-à-dire à une description/interprétation locale des gestes des praticiens alors qu'elle est d'une redoutable exigence professionnelle si l'on conduit le processus à son terme.

On ne peut faire l'économie de l'examen des discours (journalistiques, populaires, politiques, sociologiques) qui renvoient aux spécificités départementales de la Seine Saint Denis ou de Paris. Le recrutement des stagiaires des IUFM, les problématiques rencontrées dans les classes (la grande mixité sociale et parfois la ghettoïsation des certaines zones dans ce département et plus généralement dans l'académie de Créteil, au risque de les stigmatiser) sont souvent l'argument des choix opérés par l'(es) institution(s) jusque dans le détail des productions écrites que nous venons de comparer ${ }^{(10)}$.

Ces analyses confirment le bien fondé d'une analyse contrastive des formations attentive aux spécificités académiques. Dans Le déclin de l'institution, F. Dubet en décrit l'évolution et remet en cause les analyses de Durkheim et de Bourdieu, qui la conçoivent comme machine à produire des valeurs, des normes et des rôles. Aujourd'hui, on constaterait un ordre scolaire dérégulé, sa recréation continue au niveau micro, dans le cadre d'interactions où la part de subjecti-

(9) Cf. P. Delormas (2011). Le degré d'assimilation à la communauté à laquelle les acteurs ont été affectés étant difficilement évaluable, j'ai préconisé dans un autre article qu'une approche générique explicite de l'écrit professionnel soit introduite dans les contenus de formation.

(10) Un colloque organisé le $1^{\text {er }}$ et 2 avril 2011 à 1'Upec sur les «Approches pluridisciplinaires des discours sur l'école en Seine-Saint-Denis » témoigne de cette problématique. URL : http://colloque.seine-saint-denis.creteil.IUFM.fr/. 
vité ferait s'écrouler la notion de rôle. La réflexivité telle que F. Dubet la conçoit suppose à la fois des acteurs socialisés et une circulation subjective dans un système. Or la compétence à construire une expérience scolaire personnelle n'est pas également partagée et les raisons de la subjectivation sont socialement définies.

\section{Références}

ACHARD-BAyle, G. (2006) : «Pour une description linguistique du mémoire professionnel universitaire. Rapport de stage et mémoire professionnel normes, usages et représentations ", Lidil, 34, Rapport de stage et mémoire professionnel. Normes, usages et représentations, 69-83.

Altet, M. (1994) : La formation professionnelle des enseignants, Paris, PUF. - (1996) : Les compétences de l'enseignant-professionnel : entre savoirs, schèmes d'action et adaptation, le savoir-analyser, in Paquay, L., Altet, M., Charlier, E. et Perrenoud, Ph. (dir.), Former des enseignants professionnels. Quelles stratégies? Quelles compétences?, Bruxelles, de Boeck, 27-40.

Charlier, E. et Donnay, J. (2001) : «Entretien avec L. Paquay », Recherche et formation, 36, Le praticien réflexif, la diffusion d'un modèle de formation, 169-172.

ClOT, Y. (1999) : La fonction psychologique du travail, Paris, P.U.F..

CRINON, J. \& GUIGUE, M. (2002) : «Être sujet de son écriture : une analyse de mémoires professionnels ». Spirale, 29, 201-219.

- (2006) : «Écriture et professionnalisation ». Revue française de pédagogie, 156, 117-169.

Delormas, P. (2011) : «Analyse du discours institutionnel de l'école : le cas de l'écriture de soi », in Pierozak, I., Bulot, T. et Blanchet, Ph. (Dir.), Approches de la pluralité sociolinguistique. Vers quelles convergences des pratiques de recherche et d'éducation ?, Cahiers de Sociolinguistique 15, 109121.

DONAHUE, Ch. (2008) : Écrire à l'université. Analyse comparée en France et aux Etats-Unis, Villeneuve d'Ascq, Presses Universitaires du Septentrion.

DONnAY, J. et CHARLIER, E. (1990) : Comprendre des situations de formation, formation de formateurs à l'analyse, De Boeck, Bruxelles.

DonNAY, J. (2001) : «Identité narrative du futur enseignant », in Alin C., Gohier $\mathrm{C}$, Enseignant formateur : la construction de l'identité professionnelle, $\mathrm{Pa}-$ ris, L'Harmattan, Collection Education et Formation. 
Dubet, F. (2002) : Le déclin de l'institution, Paris, Seuil.

HATTON, N. \& SMITH, D. (1995) : «Reflection in teacher education : towards definition and implementation », Teaching and Teacher Education, 11(1), 3349.

LATOUR, B. (1996) : "Sur la pratique des théoriciens », in Jean-Marie Barbier. (ed.), Savoirs théoriques et savoirs d'action, Paris, PUF, 131-146.

Maingueneau, D. (1991) : L'Analyse du discours, Introduction aux lectures de l'archive, Paris, Hachette.

- (1993) : "Analyse du discours et archive ", Semen, 08, Configurations discursives, 1993, http://semen.revues.org/document4069.html.

Michaud, C. \& ALIN, C. (2011) : Le portfolio : un en-(je)u de formation et de développement professionnel. Acte du congrès international Actualité de la recherche en Education et en Formation AREF 2010 - Université de Genève, 13-16 septembre 2010. Suisse.

https://plone2.unige.ch/aref2010/communications-orales/premiers-auteurs-en-m/Le \%20portfolio.pdf/view.

Oger, C. et Ollivier-YANnIV, C. (2003) : « Conjuguer analyse du discours institutionnel et sociologie compréhensive : vers une anthropologie des discours institutionnels », Mots, Les langages du politique, 71, 125-145.

PERRENOUD, P. (1994) : La formation des enseignants entre théorie et pratique, Paris, L'Harmattan.

- (2001) : Développer la pratique réflexive dans le métier d'enseignant, Paris, ESF.

RicARD-Fersing, É., Dubant-Birglin, M.-J. et Crinon, J. (2002) : « Mémoires professionnels et portfolios dans la formation des enseignants. Une étude comparative », Revue française de pédagogie, 139, 121-129.

SCHNEUWLY, B. et DolZ, J. (1997) : «Les genres scolaires des pratiques langagières aux objets d'enseignement », Repères, 15, Pratiques langagières et enseignement du français à l'école, 27-40.

SCHÖN, D.A. (1993) : Le praticien réflexif, À la recherche du savoir caché dans l'agir professionnel, Montréal, Éditions Logiques.

VAN MANEN, M. (1977) : Linking ways of knowing with ways of being practical. Curriculum and Inquiry, 6(3), 205-228.

VelciC-CANIVEZ, M. (2006) : « Le Rapport de stage et la distanciation par rapport à soi », Lidil, 34, 49-68.

WRANA, D. (2006) : Das Subjekt schreiben, Schneider Verlag GmbH. 


\section{ANNEXE}

Tableau 1 : Les indices de réflexivité personnelle dans les écrits réflexifs de l'IUFM de Créteil

\begin{tabular}{|l|c|c|c|c|c|c|c|c|c|c|c|c|c|}
\hline \multicolumn{1}{|r}{ Écrits } & 1 & 2 & 3 & 4 & 5 & 6 & 7 & 8 & 9 & 10 & 11 & 12 & 13 \\
\hline $\begin{array}{l}\text { Indices de vue unique } \\
\text { du stagiaire }\end{array}$ & $\mathrm{X}$ & $\mathrm{X}$ & $\mathrm{X}$ & & & & & $\mathrm{X}$ & $\mathrm{X}$ & $\mathrm{X}$ & $\mathrm{X}$ & $\mathrm{X}$ & $\mathrm{X}$ \\
\hline $\begin{array}{l}\text { Ordre : narration, critique, } \\
\text { rectification, projet révisé }\end{array}$ & $\mathrm{X}$ & $\mathrm{X}$ & $\mathrm{X}$ & & & & & $\mathrm{X}$ & $\mathrm{X}$ & $\mathrm{X}$ & $\mathrm{X}$ & $\mathrm{X}$ & $\mathrm{X}$ \\
\hline $\begin{array}{l}\text { Déclaration de } \\
\text { principes et valeurs/ ethique }\end{array}$ & $\mathrm{X}$ & & & & & & & & & & & & \\
\hline $\begin{array}{l}\text { Difficultés de } \\
\text { l'enseignant et de l'élève }\end{array}$ & & & & & & & & $\mathrm{X}$ & $\mathrm{X}$ & $\mathrm{X}$ & $\mathrm{X}$ & $\mathrm{X}$ & $\mathrm{X}$ \\
\hline Hésitations/questions intimes & $\mathrm{X}$ & & $\mathrm{X}$ & & & & & & & & & $\mathrm{X}$ & $\mathrm{X}$ \\
\hline
\end{tabular}

Tableau 2: Les indices de réflexivité personnelle dans les portfolios de l'IUFM de Paris

\begin{tabular}{|c|c|c|c|c|c|c|c|c|c|c|c|c|c|}
\hline Écrits & 1 & 2 & 3 & 4 & 5 & 6 & 7 & 8 & 9 & 10 & 11 & 12 & 13 \\
\hline \multicolumn{14}{|l|}{ Pt de vue unique du stagiaire } \\
\hline $\begin{array}{l}\text { Ordre : narration, critique, recti- } \\
\text { fication, projet révisé }\end{array}$ & & & & & & & & & & $x$ & & & \\
\hline $\begin{array}{l}\text { Déclaration de principes et va- } \\
\text { leurs/ ethique }\end{array}$ & $x$ & & & & & & $x$ & $x$ & $x$ & $x$ & $x$ & $x$ & $x$ \\
\hline $\begin{array}{l}\text { Difficultés de l'enseignant et de } \\
\text { l'élève }\end{array}$ & $x$ & & & & & & $x$ & $x$ & $x$ & $x$ & $x$ & $x$ & $x$ \\
\hline Hésitations/questions intimes & & & & & & & & & & & & & \\
\hline
\end{tabular}

Tableau 3 : Les indices de scientificité dans les écrits réflexifs de l'IUFM de Créteil

\begin{tabular}{|l|l|l|l|l|l|l|l|l|l|l|l|l|l|}
\hline Indices & 1 & 2 & 3 & 4 & 5 & 6 & 7 & 8 & 9 & 10 & 11 & 12 & 13 \\
\hline Mise en scène du chercheur & & & & & & & & & & & & & \\
\hline Référence aux textes officiels & & & & & & & & & & & & & \\
\hline $\begin{array}{l}\text { Les apprentissages de l'élève } \\
\text { comme objets de savoir, } \\
\text { de recherche }\end{array}$ & & & & & & & & & X & & & & \\
\hline $\begin{array}{l}\text { Des références théoriques aux } \\
\text { leçons professionnelles }\end{array}$ & & & & & & & & & & & & & \\
\hline
\end{tabular}

Tableau 4: Les indices de scientificité dans les portfolios de l'IUFM de Paris

\begin{tabular}{|l|l|l|l|l|l|l|l|l|l|l|l|l|l|}
\hline Écrits & 1 & 2 & 3 & 4 & 5 & 6 & 7 & 8 & 9 & 10 & 11 & 12 & 13 \\
\hline Indices & & & & & & & & & & & & & \\
\hline Mise en scène du chercheur & & & & & & & $\mathrm{X}$ & & & $\mathrm{X}$ & $\mathrm{X}$ & & $\mathrm{X}$ \\
\hline Rérence aux textes officiels & & & & & & & $\mathrm{X}$ & $\mathrm{X}$ & $\mathrm{X}$ & $\mathrm{X}$ & $\mathrm{X}$ & $\mathrm{X}$ & $\mathrm{X}$ \\
\hline
\end{tabular}




\begin{tabular}{|l|l|l|l|l|l|l|l|l|l|l|l|l|l|}
\hline $\begin{array}{l}\text { Les apprentissages de l'élève } \\
\text { comme objets de savoir, de } \\
\text { recherche }\end{array}$ & & & & & & & $X$ & $X$ & $X$ & $X$ & $X$ & $X$ & $X$ \\
\hline $\begin{array}{l}\text { Des références théoriques aux } \\
\text { leçons professionnelles }\end{array}$ & & & & & & & $X$ & & & $X$ & $X$ & & $X$ \\
\hline
\end{tabular}

Tableau 5 : Les indices de professionnalité dans les écrits réflexifs de l'IUFM de Créteil

\begin{tabular}{|l|c|c|c|c|c|c|c|c|c|c|c|c|c|}
\hline Écrits & 1 & 2 & 3 & 4 & 5 & 6 & 7 & 8 & 9 & 10 & 11 & 12 & 13 \\
\hline $\begin{array}{l}\text { Indices } \\
\text { Centration/préparation de la }\end{array}$ & $\mathrm{X}$ & $\mathrm{X}$ & $\mathrm{X}$ & & & & & $\mathrm{X}$ & $\mathrm{X}$ & $\mathrm{X}$ & & $\mathrm{X}$ & $\mathrm{X}$ \\
\hline $\begin{array}{l}\text { Centration sur un problème } \\
\text { unique }\end{array}$ & $\mathrm{X}$ & $\mathrm{X}$ & $\mathrm{X}$ & & & & & $\mathrm{X}$ & & & $\mathrm{X}$ & $\mathrm{X}$ & $\mathrm{X}$ \\
\hline Pratique en situation réelle & $\mathrm{X}$ & $\mathrm{X}$ & $\mathrm{X}$ & & & & & $\mathrm{X}$ & $\mathrm{X}$ & $\mathrm{X}$ & $\mathrm{X}$ & $\mathrm{X}$ & $\mathrm{X}$ \\
\hline Référence théorique/biblio & $\mathrm{X}$ & & & & & & & & & & & & $\mathrm{X}$ \\
\hline Annexes & & & & & & & & & & & & & \\
\hline
\end{tabular}

Tableau 6: Les indices de professionnalité dans les portfolios de Paris

\begin{tabular}{|l|l|l|l|l|l|l|l|l|l|l|l|l|l|}
\hline \multicolumn{1}{|c|}{ Écrits } & 1 & 2 & 3 & 4 & 5 & 6 & 7 & 8 & 9 & 10 & 11 & 12 & 13 \\
\hline $\begin{array}{l}\text { Indices } \\
\text { classe et décisions }\end{array}$ & & & & & & & & $\mathrm{X}$ & $\mathrm{X}$ & $\mathrm{X}$ & $\mathrm{X}$ & $\mathrm{X}$ & $\mathrm{X}$ \\
\hline $\begin{array}{l}\text { Centration sur un problème } \\
\text { unique }\end{array}$ & & & & & & & & & & & & & \\
\hline Pratique en situation réelle & & & & & & & & $\mathrm{X}$ & $\mathrm{X}$ & $\mathrm{X}$ & $\mathrm{X}$ & $\mathrm{X}$ & $\mathrm{X}$ \\
\hline Référence théorique/biblio & & & & & & & & & & & & & $\mathrm{X}$ \\
\hline
\end{tabular}

